\title{
The puzzling origin and evolution of stellar populations in $\omega$ Centauri
}

\author{
A. Sollima ${ }^{1} \dagger$, F. R. Ferraro ${ }^{1}$, M. Bellazzini ${ }^{2}$ \\ and E. Pancino ${ }^{2}$ \\ ${ }^{1}$ Dipartimento di Astronomia, Università di Bologna \\ via Ranzani 1, 40127-I Bologna, Italy \\ 2 INAF Osservatorio Astronomico di Bologna \\ via Ranzani 1, 40127-I Bologna, Italy
}

\begin{abstract}
On the basis of large photometric and spectroscopic datasets, we investigated the general properties of the different sub-populations of $\omega$ Centauri in different regions of the colormagnitude diagram. In particular, we analysed the morphology, the structure and the chemical properties of the stellar populations of the system in variuos evolutionary stages. Such a large observational effort allowed us to constraint the different hypotesis on the origin and chemical evolution of this peculiar stellar system.
\end{abstract}

Keywords. methods: data analysis, techniques: photometric, techniques: spectroscopic, stars: abundances, stars: evolution, stars: Population II, globular clusters: $\omega$ Centauri

We present the main resilts of a large program devoted to the investigation of the origin and evolution of the stellar system $\omega$ Centauri (see Ferraro et al. 2003). We used a large photometric and spectroscopic dataset obtained with the last generation of imagers and spectrometers mounted at the ESO telescopes and on board HST to investigate the general properties of the different sub-populations observed in $\omega$ Cen in different regions of the color-magnitude diagram (CMD).

Using optical BVI photometry with FORS1 at the VLT in the central region of the cluster we confirmed the existence of multiple stellar populations along the red giant branch (RGB). Five separate components with different metallicities and relative frequencies have been identified (Sollima et al. 2005a). The discrete structure of the RGB indicates that $\omega$ Cen experienced several bursts of star formation separated in time producing stellar populations with different metal content.

An accurate analysis of the subgiant branch (SGB) structure, performed using deep HST/ACS photometry and a spectroscopic survey obtained with FLAMES at the VLT, allowed us to derive the relative ages of the different sub-populations of the cluster. The ages derived for the different stellar populations of the system turn out to be all comparable within 2 Gyr, regardless of any choice of helium abundance (Sollima et al. 2005b). This result excludes an extended star formation period for this stellar system.

From a deep photometric survey performed with FORS1at the VLT we sampled the main sequence (MS) population of $\omega$ Cen over the entire radial extent of the cluster. Thanks to this analysis we were able to detect the bifurcation in the MS of the system (already observed by Bedin et al. 2004) on a large field of view up to 26 ' from the cluster center (see Fig. 1, Sollima et al. 2007). The comparison between the radial distribution of the blue MS component (bMS) stars with that of the other "normal" MS stars indicates that the bMS is more concentrated toward the cluster center. The location of the bMS in

\section{$\dagger$ email: antonio.sollima@bo.astro.it}




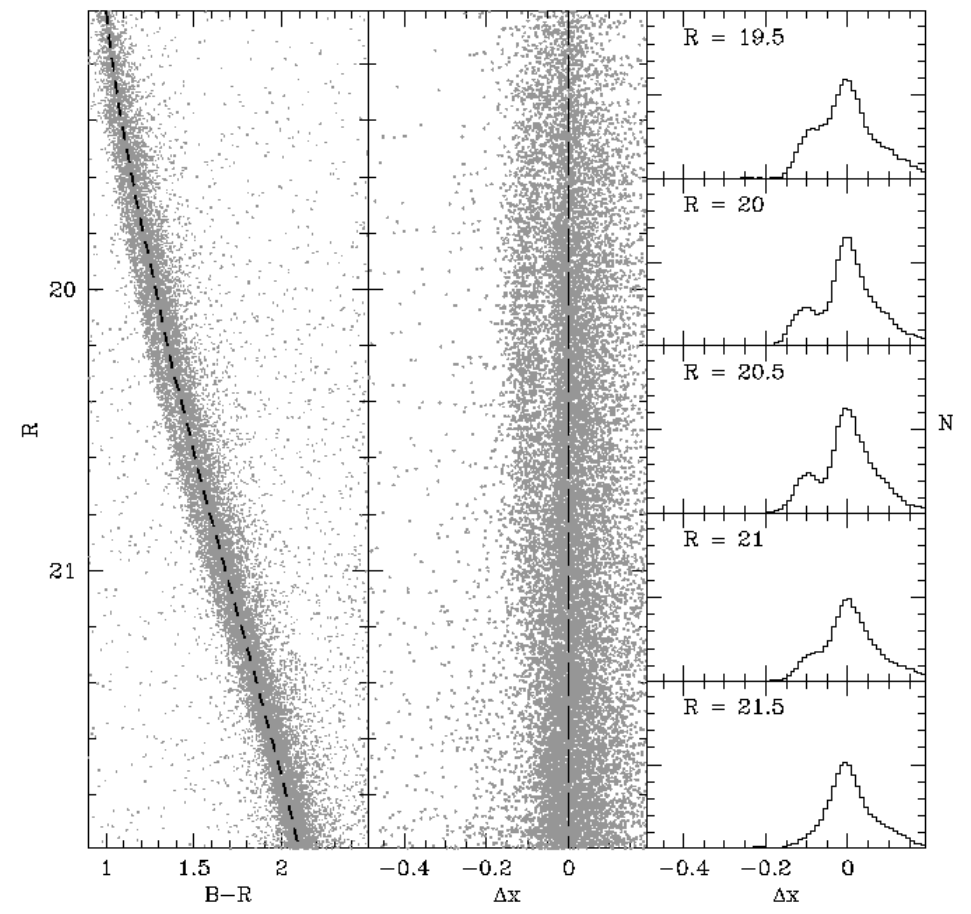

Figure 1. The zoomed (R, B-R) CMD of $\omega$ Cen in the bMS region is shown in the left panel. The mean ridge line of the rMS population is overplotted to the CMD as a dashed line. The distribution of MS stars with respect to the reference mean ridge line is shown in the middle panel. In the right panel the histograms of the distances from the mean ridge line at different magnitude levels are shown.

the CMD can be explained by assuming a large helium overabundance $(\mathrm{Y} \sim 0.4$ ) for the stars belonging to this population. However, the spectroscopic observation of a sample of 74 RR Lyrae confirmed the presence of a metal-intermediate $([\mathrm{Fe} / \mathrm{H}] \sim-1.2)$ RR Lyrae population in $\omega$ Cen with luminosity and pulsational properties that are incompatible with any helium overabundance (Sollima et al. 2006). This evidence suggests the existence of two stellar populations with similar metallicities but very different helium abundances that seems to coexists within the cluster.

\section{References}

Bedin, L. R., Piotto, G., Anderson, J., Cassisi, S., King, I. R., Momany, Y. \& Carraro, G. 2004, ApJ 605, L125

Ferraro, F. R., Bellazzini, M., Origlia, L., Pancino, E. \& Sollima, A. 2003, in: "New Horizons in Globular Cluster Astronomy", Piotto, G. Meylan, S. G. Djorgovski \& M. Riello (eds.), (ASP Conf. Ser.), vol. 296, p. 215

Sollima, A., Ferraro, F. R., Pancino E., \& Bellazzini, M. 2005a, MNRAS 357, 265

Sollima, A., Pancino E., Ferraro, F. R., Bellazzini, M., Straniero, O. \& Pasquini, L. 2005b, ApJ 634,332

Sollima, A., Borissova, J., Catelan, M., Smith, H. A., Minniti, D., Cacciari, C. \& Ferraro, F. R. 2006 ApJ 640, L43

Sollima, A., Ferraro F.R., , Bellazzini, M., Origlia, L., Straniero, O., \& Pancino, E. 2007, ApJ 654,915 